\section{STUDY OF THE IONOSPHERE DURING SOLAR ECLIPSES}

Solar Eclipses and the lonosphere

A Symposium held under the auspices of the International Council of Scientific Unions Mixed Commission on the Ionosphere, in London in August 1955. Edited by W. J. G. Beynon and G. M. Brown. (Vol. 6 of Special Supplements to the Journal of Atmospheric and Terrestrial Physics.) Pp. $x+330$. (London and New York: Pergamon Press, 1956.) 140s.; 21 dollars.

$\mathrm{W}$

HILE it has long been known that the Sun is the primary cause of the existence of the ionosphere, there has been some controversy as to the exaet mechanism by which the various layers which reflect radio waves are ionized. Much information has been accumulated concerning the diurnal variation of conditions in the ionosphere, and the results have been used in a critical examination of the processes of ionization and recombination in the reflecting layers. An eclipse of the Sun clearly provides an excellent opportunity of investigating these processes over a short interval of time, and particularly of studying the relative contributions of radiation from the Sun's disk and from the corona. For these reasons every opportunity is sought by radio-physicists to observe the changes occurring in the ionosphere during an eclipse, especially when the path of totality is accessible and the time is not too near either sunrise or sunset, when the eclipse effects might be masked by the normal daily changes.

In August 1955, certain aspects of eclipse phenomena were discussed at an international symposium organized by the Mixed Commission on the Ionosphere and held at the Royal Society (see Nature, 176, 947. 1955). This symposium was attended by delegates from eighteen countries, and the volume under review contains an account of its proceedings, with the fifty-three formal papers reproduced in full, and brief accounts of the accompanying discussions. The material is presented in nine sections beginning with an introductory survey of "Solar Eclipses and the Ionosphere" by Mr. J. A. Ratcliffe. The second section comprises three theoretical papers on the subject ; and this is followed by the third and largest section in which some nineteen papers describe the results obtained in ionospheric observations during recent, solar eclipses. Detailed consideration is given in the next two sections to the effect of solar radiation on the ionosphere in conjunction with the processes of recombination, which are surveyed and discussed. The sixth section is devoted to the relationship between geomagnetic occurrences and eclipse phenomena, introduced by a survey by Prof. S. Chapman. In the past decade, the radio astronomer has developed a new tool for studying solar radiation, and the seventh section contains papers describing observations made at short radio wave-lengths in seven countries. After a collection of miscellaneous papers, the volume concludes with a summary in which attention is directed to the evidence, first that the ionizing radiation from the Sun is not uniformly spread over the visible disk, and secondly, even when the disk is totally eclipsed, there is still some 10-15 per cent of residual ionizing radiation in operation.

The production of the work is excellent in every way; it is printed on good quality paper and lavishly illustrated with very clear diagrams and some unusually good reproductions of ionospheric records. The editors, Drs. W. J. G. Beynon and G. M. Brown, have prepared all the papers in as concise a form as possible, and provided a short abstract at the head of each contribution. They have also added a bibliography of all papers on the subject published between 1912 and 1954, arranged chronologically and provided with an author index. This is probably the most comprehensive collection of references in existence dealing with the effects of solar eclipses on the ionosphere, and is a most useful addition to the papers presented at the symposium.

The future research workers and students of this subject are indeed fortunate to have this comprehensive work of reference available, and both the editors and publishers are to be congratulated on its successful production. $\quad$ R. L. SMITH-Rose

\section{FIELD THEORY AND MESON PRACTICE}

Mesons and Fields

Vol. 1 : Fields. By Prof. Silvan Schweber, Prof. Hans A. Bethe and Frederic de Hoffmann. Pp. xvi+449. Vol. 2 : Mesons. By Prof. Hans A. Bethe and Frederic de Hoffmann. Pp. xiii + 446. (Evanston, Ill. : Row, Peterson and Company, 1955.) 8 dollars each volume; 15 dollars the set of 2 volumes.

$\mathrm{T}$ is a reflexion on the present state of physics that in preparing a book on mesons and fields the authors were led to produce one volume on fields and another on mesons, with one of the authors essentially concentrating on one volume, and the other two on the other, with only a very tenuous connexion between the two parts.

Vol. I therefore becomes a text-book on field theory, and on closer inspection it is really a textbook on quantum electrodynamics. The reason for this is that the volume concentrates almost completely on the solution of quantum field equations by series in powers of the coupling constant, which is indeed the only procedure that can to-day be applied consistently to give unambiguous answers. It has been very successful in describing the interaction of electrons with the electromagnetic radiation, but it fails completely in the case of meson theory because the coupling constant there is known to be so large that a power series would be practically useless even if it converged (in fact, it is almost certainly divergent). This failure is evident theoretically and also confirmed empirically since the data are in clear contradiction with the results of perturbation theory, a fact stressed clearly in the second volume but apparently not mentioned in the first. Indeed, the statement in the introduction (p. xiv), "We therefore believe that renormalization theory should be considered as substantially correct, and applied to meson theory", may easily induce the reader to think that the methods developed in the volume may be applied directly to the meson field. The remark is probably intended in a more general sense, looking for an extension of the general principles of renormalization to strong interactions, but this is as yet only a very vague programme.

Non-perturbation solutions are mentioned in section 25, but the only results obtained there relate to equations of the Bethe-Salpeter type which go 